\title{
CARACTERÍSTICAS DEL INGLÉS DE LOS TÉRMINOS ECONÓMICO- FINANCIEROS: UN ENFOQUE TRADUCCIONAL CHARACTERISTICS OF THE ENGLISH ECONOMIC AND FINANCIAL TERMS: A TRANSLATIONAL APPROACH María Soledad Cruz Martínez María Luisa Cruz. Vargas \\ Universidad de Almería
}

\section{ABSTRACT}

Nowadays the translation from English to Spanish of financial terms has focused, mainly, on the terms usually used in the markets, due to the great importance that these institutions have acquired inside the economic systems. Nevertheless, there exists a field of Finance, neighbouring Economic Theory, which provides few corpuses so developed as the first ones. For this reason, we will begin by dealing in depth with the general characteristics of the English financial terms. This classification will be our framework in order to achieve the two aims of this paper. Firstly, this paper tries to detect the needs of translation of the most abstract financial terms; and, secondly, it attempts to offer a proposal of translation of them, or a wider information about the existing ones. To accomplish such purposes, this work lies on the translational current of Terminology and tries to identify some gaps in the financial English which require a certain intervention.

KEYWORDS: Terminology, Translation, Finance, financial market

\section{RESUMEN}

En la actualidad, la traducción al español de los términos financieros anglosajones se ha centrado, fundamentalmente, en aquellos que se utilizan habitualmente en los mercados, debido a la gran importancia que estas instituciones han adquirido dentro de los sistemas económicos. No obstante, existe una parte de las Finanzas, la más próxima a la Teoría Económica, que no cuenta con unos corpus tan desarrollados como los primeros. Por ello, comenzaremos estudiando en profundidad las características generales de los términos financieros en inglés. Esta clasificación nos servirá como marco de referencia para el cumplimiento de los dos objetivos de este artículo. En primer lugar, pretendemos detectar las necesidades de traducción de los 
términos financieros más abstractos; $y$, en segundo lugar, ofrecer una propuesta de traducción de los mismos o de mayor información para los ya existentes. Para ello, este trabajo se sitúa en la corriente traduccional de la Terminología y pretende identificar algunas lagunas en el inglés financiero que requieren cierta intervención.

PALABRAS CLAVE: Terminología, Traducción, Finanzas, mercado financiero

\section{INTRODUCCIÓN}

En la actualidad, la traducción al español de los términos financieros procedentes del inglés se ha centrado, fundamentalmente, en aquellos que se utilizan habitualmente en los mercados, debido a la gran importancia que estas instituciones han adquirido dentro de los sistemas económicos mundiales. Con este objetivo y como paso previo para ofrecer una propuesta de traducción de dichos términos, ha sido necesario llevar a cabo un estudio general de las características léxico-semánticas de los términos financieros en inglés, siendo el referente principal en este estudio el Diccionario de Términos Económicos, Financieros y Comerciales (Inglés-Español, Spanish-English) de Alcaraz Varó y Brian Hughes (2008).

Ahora bien, aunque la traducción de muchos de estos términos en los diccionarios es correcta, su explicación desde un punto de vista financiero contribuiría a la clarificación de su significado y uso, lo que sería de vital importancia para el trabajo del traductor. Dicho de otra forma, nuestra propuesta es que los diccionarios de términos financieros deberían ofrecer una explicación, además de la traducción, del término. Por otra parte, existen otros términos financieros que, en el mundo anglosajón, se han desarrollado como consecuencia de la proliferación de los mercados de productos derivados y de los modelos matemáticos que tratan de explicarlos.

Así, Hernández y Hernández (2008: 81-108) señalan que algunos artículos (véase, por ejemplo, Neff Van Aertselaer, 2006) distinguen entre diversos géneros, pero que raramente se distinguen diferentes subáreas dentro de un mismo género, siendo éste el caso de la Economía . Por ello, los autores antes mencionados proponen en su trabajo un análisis estructural, gramatical, metatextual, y retórico de las siguientes cuatro subáreas dentro de la Economía: Economía Aplicada, Economía Cuantitativa, Economía Financiera, y Administración y Gestión Empresarial. De ellas, la que más se identifica con el objeto de nuestro estudio es la de Economía Cuantitativa aunque, para su investigación, utilizan solamente dos artículos lo que, en nuestra opinión, es una representación muy poco significativa. 
Para tratar de cubrir este déficit metodológico, nuestro trabajo aborda el estudio de las características y el problema de la traducción de aquellos términos financieros que se sitúan en la confluencia de la Economía, las Finanzas y las Matemáticas, tal y como se ilustra en la figura 1. En este sentido, nos situamos en un ámbito de la Traducción especializada que puede ser de gran utilidad para los profesionales de las finanzas más cuantitativas.

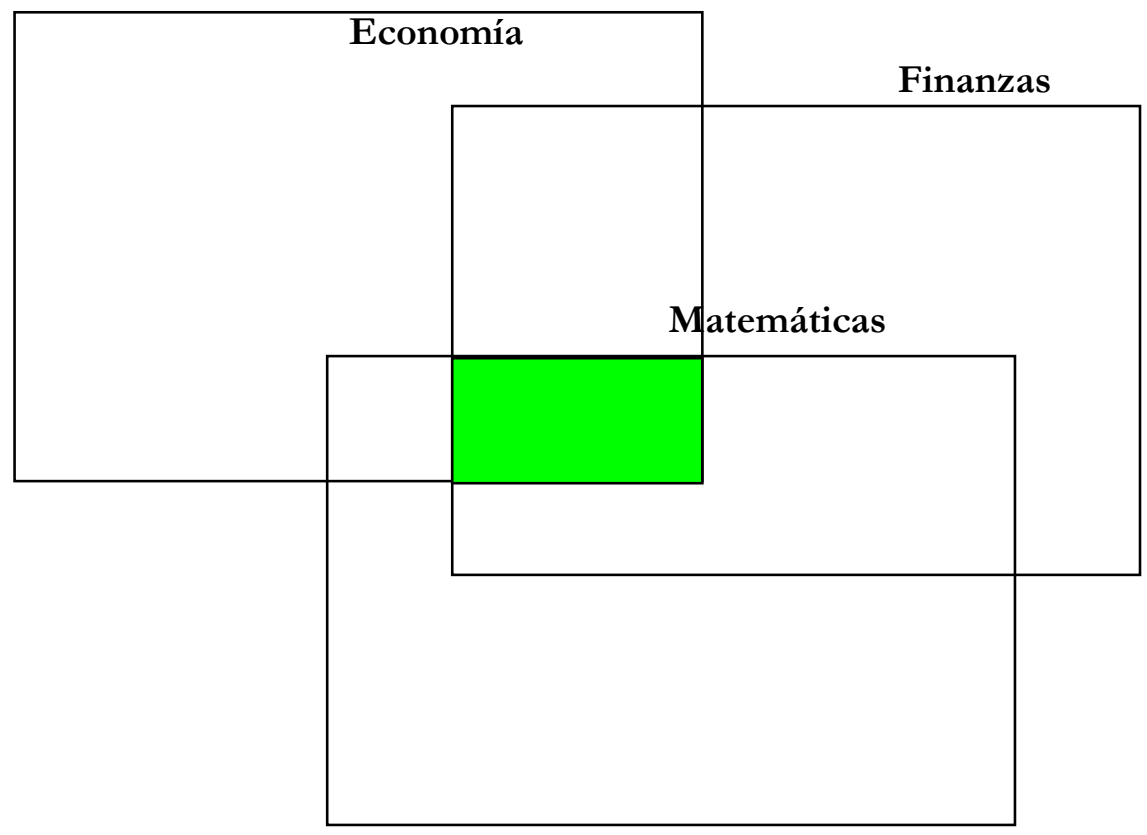

Figura 1: Ámbito de la investigación. Fuente: Elaboración propia.

Para ello, hemos seleccionado una serie de términos pertenecientes a la intersección de las tres disciplinas mencionadas en el párrafo anterior, asegurándonos, en la medida de lo posible, de que no aparecían en los diccionarios existentes hasta la fecha, o bien de que el término propuesto en español no estaba justificado plenamente. Para ello, hemos utilizado el corpus contenido en diversas publicaciones de referencia en esta materia (algunas de ellas están reseñadas en la bibliografía final), a saber:

1. Diccionarios técnicos.

2. Diccionarios en línea.

3. Artículos científicos.

4. Papeles de trabajo (working papers).

5. Informes financieros.

6. Páginas Web. 
Así pues, abordando en profundidad el estudio de las características del inglés de los textos financieros (en particular, el léxico, la semántica, la metáfora, la prosodia y los neónimos), los objetivos que pretende alcanzar este trabajo son los siguientes:

1. Llevar a cabo una selección de términos financieros procedentes de la Economía Cuantitativa, o de aquellos en los que pueda existir alguna carencia en los diccionarios especializados. Para ello, comprobaremos si la traducción de los términos financieros escogidos incluye consideraciones en torno a las características, propiedades y modelo de funcionamiento dentro de sus respectivos mercados.

2. Proponer soluciones concretas a la traducción de los términos financieros seleccionados.

3. Complementar la traducción de determinados términos financieros atendiendo a las características inherentes al propio concepto financiero.

La motivación de este trabajo se encuentra fundamentada en la literatura especializada, donde algunos autores defienden el papel de los traductores en la generación de los términos (Fijo León, 2007: 193-198). En una concepción más amplia, los traductores pueden participar en los procesos de construcción terminológica, ya que tienen a su alcance diferentes posibilidades denominativas de entre las cuales deben seleccionar las más adecuadas en función de las circunstancias temáticas, pragmáticas y contextuales. Es más, cuando exista un vacío denominativo que los glosarios y diccionarios no han cubierto, los traductores de determinadas lenguas y en determinadas situaciones se convierten en creadores de términos. Esto justifica que necesiten una buena formación en Terminología y Neología. Para dar respuesta a dicho objetivo, este artículo debe situarse, como ya hemos anticipado, dentro de la corriente traduccional de la Terminología, pretendiendo un acercamiento de las Finanzas al campo de la Traducción especializada.

Por lo que respecta a la organización de este trabajo, en la Sección 2 se ha puesto de manifiesto la necesidad de estrechar la relación entre la Terminología y la Traducción. A continuación, en la Sección 3, se ha llevado a cabo un trabajo de documentación de un conjunto de términos financieros que se sitúan en la confluencia de la Economía, las Finanzas y las Matemáticas. Para ello, hemos seguido la metodología presentada por Cabré y Tebé (2005: 19-41) con el objetivo de conseguir una traducción de los términos propuestos de acuerdo con los corpus disponibles. Por último, la Sección 4 presenta las principales conclusiones de este trabajo. 
Como es sabido, la Terminología es una disciplina que se estructura en torno a las unidades terminológicas. Si la consideramos desde esta perspectiva, la Terminología se considerará como una materia auxiliar de la Traducción. Ahora bien, en relación con la Traducción, se contemplan tres posibilidades de implicación del traductor según el nivel de actividad que quiera asumir en Terminología (Cabré, 2008b):

- El traductor como usuario de la Terminología, que se limita a emplear las soluciones que los diccionarios o los servicios de consulta le proporcionan.

- El traductor como terminólogo puntual, que resuelve los problemas que se encuentra cuando lleva a cabo una traducción.

- El traductor como gestor de Terminología formando parte de redes cooperativas de traductores en general o de la misma especialidad.

Evidentemente, nuestro trabajo pretende situarse en el nivel de la tercera actividad señalada. Pero, por parte de los traductores, hay una serie de creencias previas (prejuicios) sobre la Terminología de los que destacaremos tres (Cabré, 2008a: 9-36):

- Para muchos traductores, la utilidad de la Terminología se limita al simple abastecimiento de equivalencias.

- Los traductores tienen una cierta desconfianza en los terminólogos como formadores de traductores.

- Los traductores desconocen la Terminología como disciplina, su método de trabajo y también sus posibles funciones en la formación de los traductores.

Sin embargo, como se ha puesto de manifiesto al principio de esta sección, no podemos estar de acuerdo con la tercera de las creencias expuestas.

La Terminología, además de tener propiedades cognitivas, es una herramienta metacognitiva que permite reforzar la formación de base de todo especialista: leer textos, comprenderlos, representar la estructura de su contenido, detectar términos, extraerlos, definirlos, buscar contextos de uso adecuados, relacionarlos entre sí, etc.

El trabajo que presentamos presta atención a "las tareas de identificación de los sectores especializados nuevos o recientes y $[\ldots]$ de hallazgo de lagunas denominativas que requieren intervención" (Díaz Hormigo, 2007) y a los posibles neologismos que pudieran surgir, tal y como queda representado en la figura 2 . 


\section{Paradigma de la pragmática Terminología (corriente traduccional)}

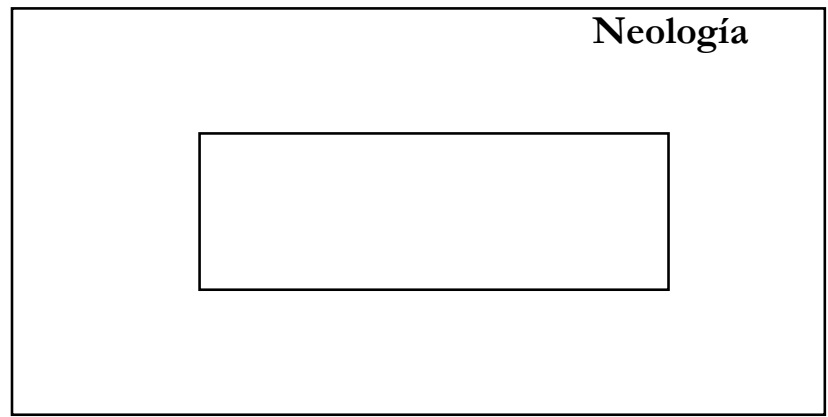

Figura 2: Marco de la investigación. Fuente: Elaboración propia.

\section{ANÁLISIS DE LOS TÉRMINOS FINANCIEROS EN INGLÉS}

\subsection{CARACTERÍSTICAS GENERALES}

Antes de comenzar con detalle el desarrollo de este epígrafe, nos gustaría puntualizar algunas características del Inglés Empresarial, lo que pensamos que puede ser de gran interés para el trabajo de preparación de los traductores en este dominio del conocimiento. Según Fuertes-Olivera y Gómez-Martínez (2004: 163-180), tanto la Economía como las Ciencias Empresariales son ejemplos de disciplinas que, requieren un cierto conocimiento de carácter cultural. Por este motivo, se da la circunstancia de que los investigadores del inglés utilizado en las empresas se enfrentan al dilema de decidir si existen unas características generales de lo que se llamaría "Inglés Empresarial". Antes de nada, nos gustaría puntualizar que, para su estudio, es necesario tener en cuenta los siguientes aspectos:

- El Inglés Empresarial es una de las variedades del IFE (Inglés para Fines Específicos) con más desarrollo.

- La investigación en este campo se está dedicando a una conciencia intercultural.

Los términos financieros no han sido abordados en profundidad desde una perspectiva léxico-semántica (Suau, 1999). No obstante, es de rigor citar uno de los trabajos lexicográficos más recientes y relevantes realizados en 
nuestro país: el Diccionario de Términos Económicos, Financieros y Comerciales de Alcaraz Varó y Hughes (2008). Los autores afirman que los términos económicos son vistos como un registro más formal y académico que los términos financieros, aduciendo la gran cantidad de raíces latinas que encontramos en ellos, por ser la Economía una disciplina académica, cuyo origen y lenguaje proviene de la tradición clásica (Alcaraz Varó y Hughes, 2008: vii). A pesar de hallar términos de origen anglosajón como clawback (recuperación, capacidad de reacción), upturn/downturn (repunte, caída o bajón), existe gran abundancia de términos de raíz latina como adverse selection (antiselección o selección adversa), moral hazard (riesgo moral), multiple correlation coefficient (cociente o coeficiente de correlación múltiple), arc elasticity of demand (elasticidad-arco de la demanda), command economy (economía autoritaria), etc.

Los términos financieros, sin embargo, presentan una etimología bien distinta. Alcaraz Varó y Hughes (2008: viii) hacen una extensa exposición de estos términos, resaltando, como principal característica, el tono coloquial de la mayoría de palabras, junto a su etimología anglosajona. Se encuentran fácilmente expresiones de registro coloquial y familiar como firms in the sector have beefed up their prices (las empresas del sector han pegado una subida brutal a los precios) o buck the market (pegarle una sacudida al mercado, oponerse a la tendencia del mercado).

En nuestra opinión, este comentario sobre las características diferenciales del registro de los términos económicos y financieros requiere una aclaración. La Economía, en general, y las Finanzas, en particular, son disciplinas que admiten tratamientos diferentes, dependiendo del emisor y del medio en que se publica. Así, por ejemplo, las Finanzas tienen conexión con las Matemáticas (más concretamente, con el Cálculo de Probabilidades y la Estadística) y con la propia Economía general, lo que hace que, en muchos casos, el registro del lenguaje financiero sea incluso más formal que el de los términos económicos.

A continuación, la tabla 1 resume las características generales del inglés financiero, basándonos para ello en la clasificación de Alcaraz Varó y Hughes (2008), aportándose además diversos ejemplos. Obsérvese que, en esta tabla, aparece la traducción al español y la explicación (columna de la derecha) de algunos términos en inglés (columna de la izquierda). El valor añadido de esta tabla es que ofrece ejemplos que no aparecen en el diccionario de Alcaraz Varó y Hughes u otros que, apareciendo en esta fuente, acompañan una explicación complementaria útil para el traductor. No obstante, otros términos aparecen sin traducción ni explicación y ello se debe a que, también en nuestro idioma, se usa la voz anglosajona. 


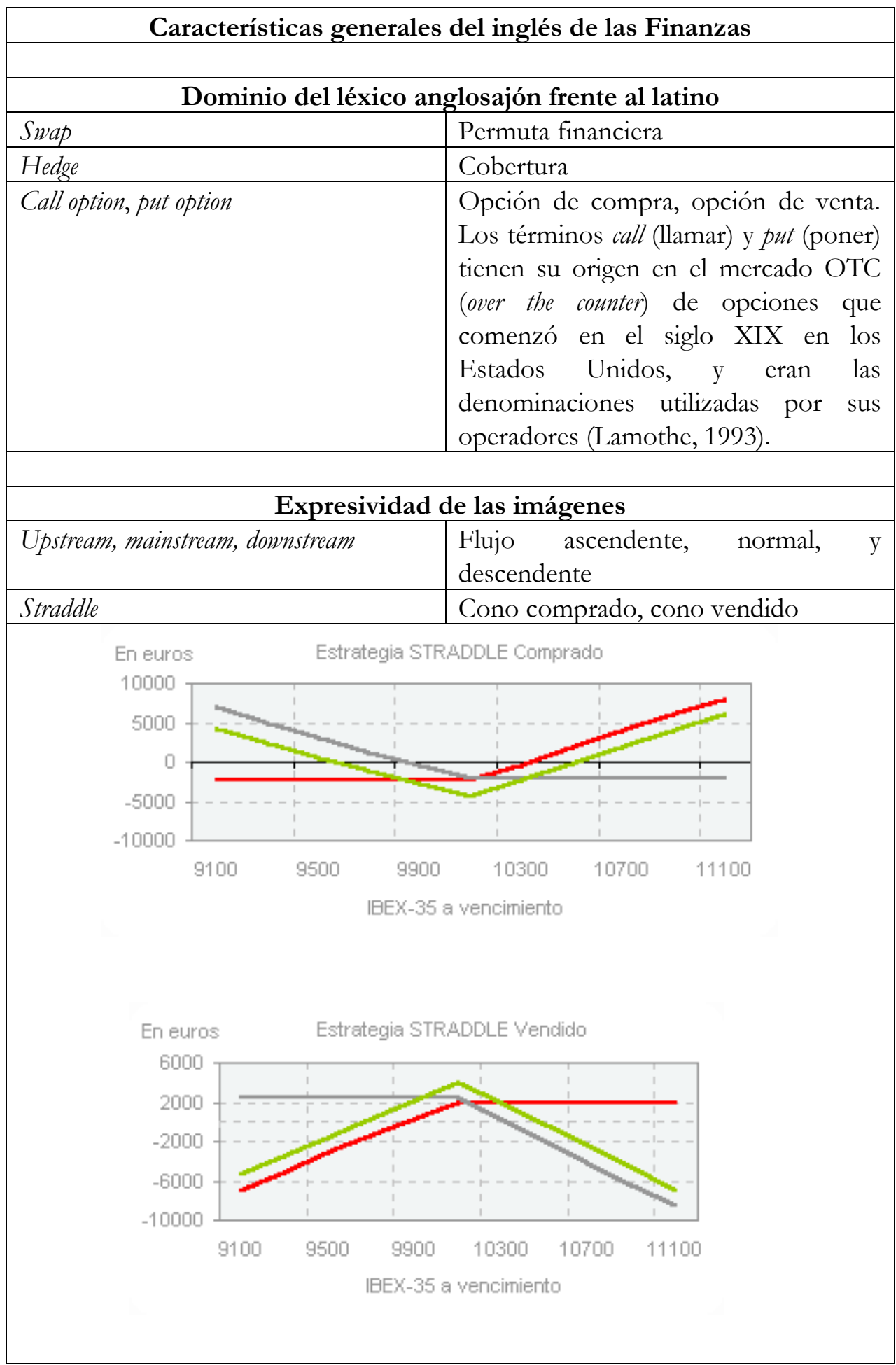


ENTRECULTURAS Número 6. ISSN: 1989-5097. Fecha de publicación: 29-01-2014

Las figuras muestran los beneficios obtenidos con la combinación conocida como straddle. Si el precio de la acción es similar al precio de ejercicio al vencimiento de las opciones, el cono produce una pérdida (resp. beneficio). Sin embargo, si hay un movimiento suficientemente grande en cualquier dirección, resultará un beneficio (resp. pérdida) significativo (Hull, 2002: 240241).

\begin{tabular}{l|l}
\hline Strangle & Cuna comprada, cuna vendida
\end{tabular}

\section{En euros $\quad$ Estrategia STRANGLE Comprado}

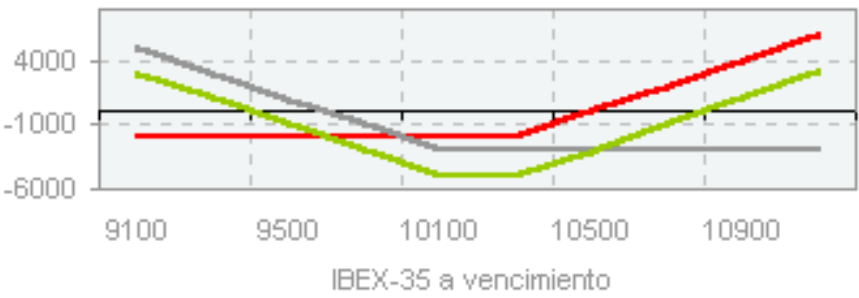

En euros $\quad$ Estrategia STRANGLE Vendido

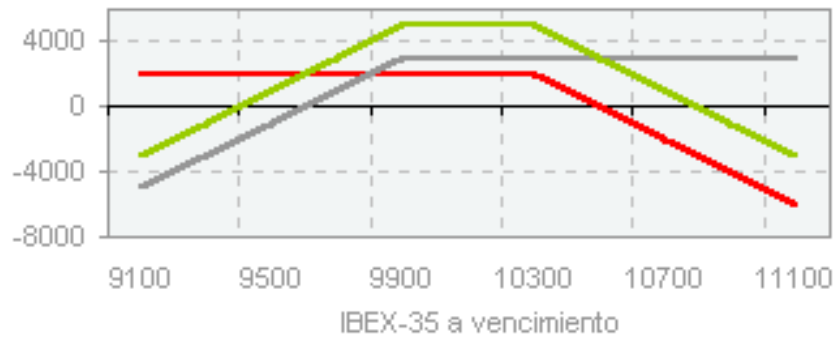

Las figuras muestran los beneficios obtenidos con la combinación conocida como strangle. Si el precio de la acción es similar al precio de ejercicio al vencimiento de las opciones, la cuna produce una pérdida (resp. beneficio) con una probabilidad mayor que en el cono. Sin embargo, si hay un movimiento suficientemente grande en cualquier dirección, resultará un beneficio (resp. pérdida) significativo (Hull, 2002: 242-243) con probabilidad menor que en el caso del cono.

\section{Lo lúdico en los juegos de palabras}

Concert party

En al primera mitad del siglo XX, un concert party era un grupo ambulante de animadores que se dedicaban a cantar y a las comedias humorísticas. Se 
ENTRECULTURAS Número 6. ISSN: 1989-5097. Fecha de publicación: 29-01-2014

\begin{tabular}{|c|c|}
\hline & $\begin{array}{l}\text { traduce por "grupo concertado de } \\
\text { inversores" }\end{array}$ \\
\hline Blue chip & $\begin{array}{l}\text { Es un término anglosajón relativo al } \\
\text { juego del póker y hace alusión a la } \\
\text { acción de una compañía industrial } \\
\text { cuyos beneficios crecen } \\
\text { continuamente y sin interrupción en el } \\
\text { pago de los dividendos. Se traduce por } \\
\text { "valores bursátiles punteros" }\end{array}$ \\
\hline \multicolumn{2}{|c|}{ Uso de siglas y formas lingüísticas mutiladas } \\
\hline $\begin{array}{l}\text { EYES (Equity Yield Enhancement } \\
\text { Securities) }\end{array}$ & EYES \\
\hline $\begin{array}{l}\text { CHIPS (Clearing House Inter-Bank } \\
\text { Payment System) }\end{array}$ & CHIPS \\
\hline $\begin{array}{l}\text { STRIPS (Separate Trading of Registered } \\
\text { Interest and Principal Securities) }\end{array}$ & STRIPS \\
\hline \multicolumn{2}{|c|}{ Uso de expresiones referidas a animales } \\
\hline Bull, bear & $\begin{array}{l}\text { Especulador alcista, especulador } \\
\text { bajista. Las denominaciones Bullish } \\
\text { (siguiendo la silueta del toro) se aplica } \\
\text { al mercado alcista, donde actúan } \\
\text { quienes prevén seguir la estrategia de } \\
\text { comprar al precio bajo más cercano y } \\
\text { vender al precio alto posterior, si se } \\
\text { cumplen las expectativas del mercado. } \\
\text { En el caso de un mercado con } \\
\text { expectativas bajistas, denominado } \\
\text { Bearish (siguiendo la silueta del oso), la } \\
\text { estrategia consiste en vender un } \\
\text { contrato de vencimiento cercano y } \\
\text { comprar otro de vencimiento posterior } \\
\text { (Costa y Font, 1992). En este sentido, } \\
\text { diferimos de la justificación aportada } \\
\text { por Alcaraz Varó y Hughes (2008: xiii) } \\
\text { cuando afirma que "el uso de la } \\
\text { palabra bear en el contexto de 'bajista', } \\
\text { etc., probablemente venga del dicho } \\
\text { inglés selling the bearskin before catching the } \\
\text { bear, es decir, 'vender la piel del oso }\end{array}$ \\
\hline
\end{tabular}


ENTRECULTURAS Número 6. ISSN: 1989-5097. Fecha de publicación: 29-01-2014

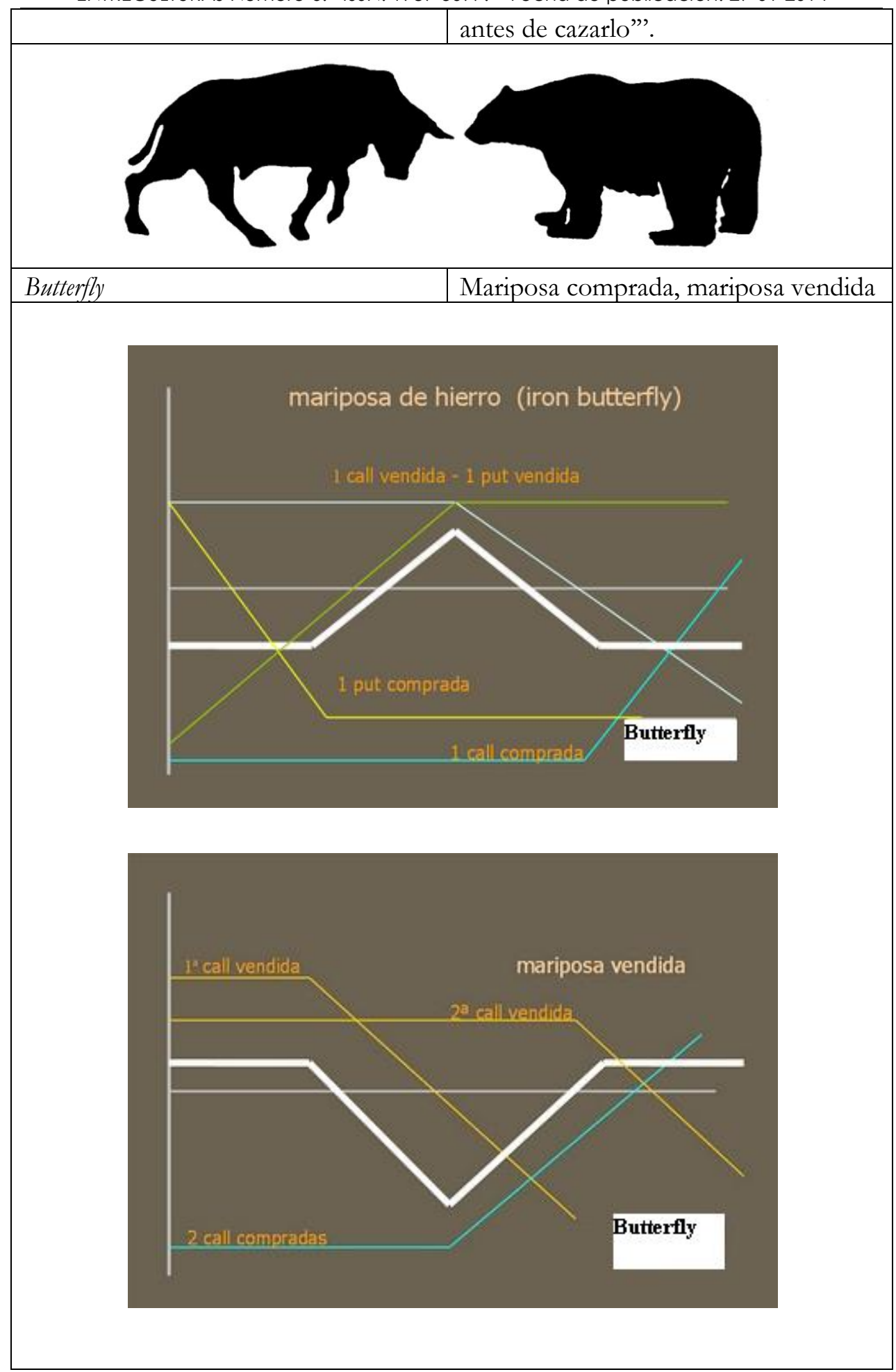


ENTRECULTURAS Número 6. ISSN: 1989-5097. Fecha de publicación: 29-01-2014 Las figuras muestran los beneficios obtenidos con la combinación conocida como butterfly. La mariposa comprada (resp. vendida) apuesta por una baja (resp. alta) volatilidad en el mercado (Lamothe, 1993: 247-248).
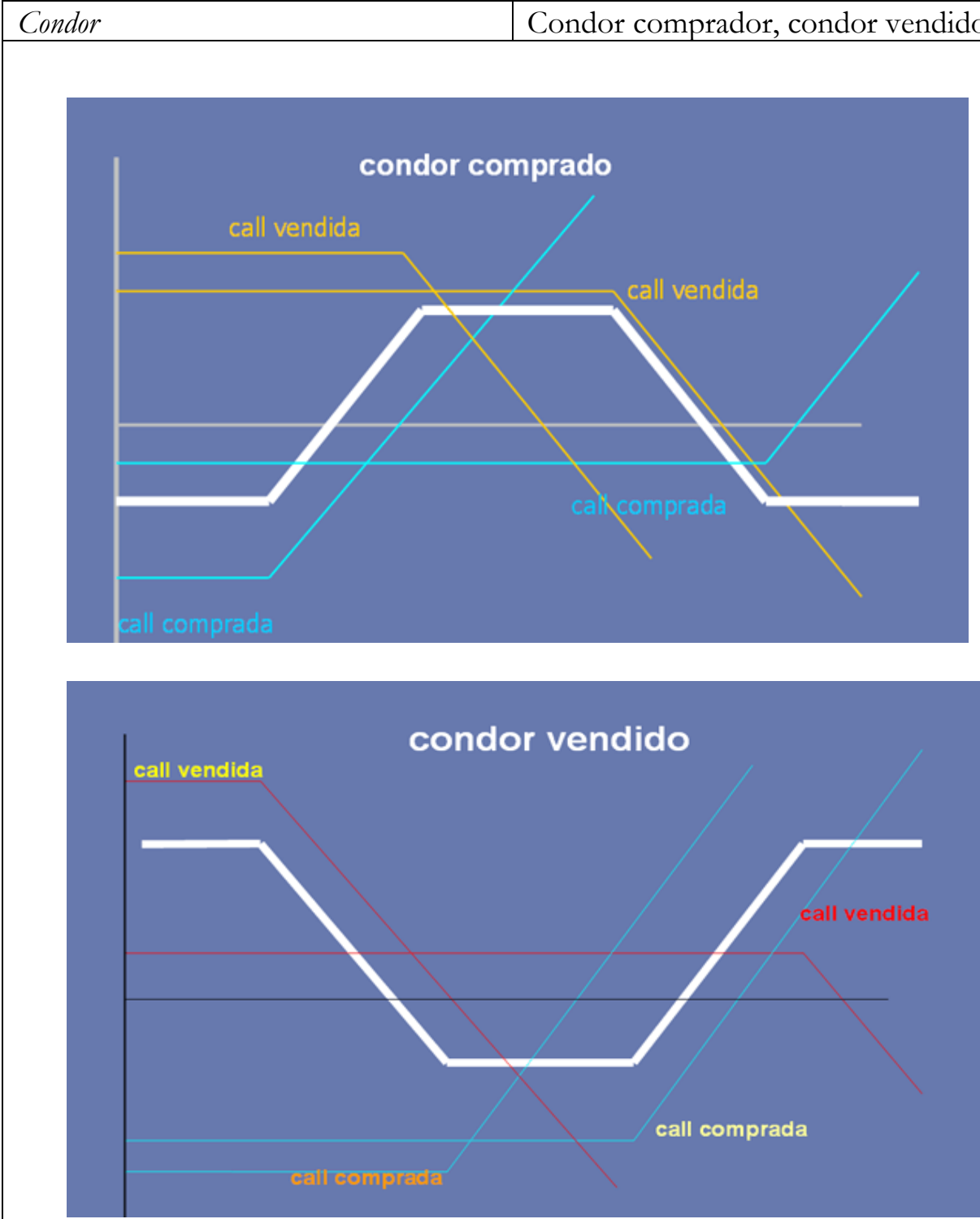

Las figuras muestran los beneficios obtenidos con la combinación conocida como condor. El cóndor comprado (resp. vendido) apuesta por una baja (resp. alta) volatilidad en el mercado (Lamothe, 1993: 249-250), más baja (resp. alta) que en el caso de la mariposa. libras esterlinas, pero emitido por un 
ENTRECULTURAS Número 6. ISSN: 1989-5097. Fecha de publicación: 29-01-2014

\begin{tabular}{|c|c|}
\hline & prestatario no británico) \\
\hline \multicolumn{2}{|c|}{ Uso de adjetivos de significado atenuador } \\
\hline Naked bond, naked debenture & $\begin{array}{l}\text { El efecto financiero o crediticio carece } \\
\text { de caución o garantía }\end{array}$ \\
\hline Nil basis & $\begin{array}{l}\text { Base cero (método de cálculo de las } \\
\text { ganancias por acción que supone que } \\
\text { no hay reparto de beneficios entre los } \\
\text { accionistas) }\end{array}$ \\
\hline Arbitrage-free & Libre de arbitraje (sin arbitraje) \\
\hline \multicolumn{2}{|c|}{ Uso de nombres de colores } \\
\hline White knight & $\begin{array}{l}\text { Caballero blanco (inversor que surge } \\
\text { de la nada para rescatar una compañía } \\
\text { que está a punto de caer en manos no } \\
\text { deseadas) }\end{array}$ \\
\hline Red interest & Números rojos, intereses deudores \\
\hline Greenback & Dólar \\
\hline Pink-sheet market & Mercado bursátil informal \\
\hline \multicolumn{2}{|c|}{ Uso de lenguaje muy expresivo } \\
\hline Dawn raid & Ataque sorpresa \\
\hline Bed and breakfasting & $\begin{array}{l}\text { Práctica existente en el Reino Unido de } \\
\text { vender títulos el último día del año } \\
\text { fiscal en curso y comprarlos de nuevo } \\
\text { al día siguiente (primer día del } \\
\text { siguiente año fiscal) }\end{array}$ \\
\hline Bells and whistles & $\begin{array}{l}\text { Cascabeles (pequeños extras que se } \\
\text { añaden a los productos financieros } \\
\text { básicos) }\end{array}$ \\
\hline Junk bond & $\begin{array}{l}\text { Bono basura (bono emitido por una } \\
\text { compañía norteamericana cuya } \\
\text { clasificación de solvencia está por } \\
\text { debajo del nivel de inversión) }\end{array}$ \\
\hline Head and shoulders & $\begin{array}{l}\text { Chart bursátil que describe cuando los } \\
\text { precios se mueven hacia arriba (brazo } \\
\text { izquierdo), luego se estabilizan } \\
\text { brevemente antes de volver a subir } \\
\text { (hombro izquierdo y cabeza). En la } \\
\text { coronilla los precios se mueven hacia }\end{array}$ \\
\hline
\end{tabular}


Tabla 1. Características léxicosemánticas del inglés financiero.

Fuente: Alcaraz Varó y Hughes (2008). Elaboración propia.

En este mismo epígrafe, nos gustaría complementar la información aportada por Alcaraz y Hughes en el sentido de que el término straddle (cono) se llama así porque la estrategia especulativa mixta consistente en la compra o venta simultánea de opciones call y put con el mismo vencimiento y precio de ejercicio, da lugar a una gráfica para los beneficios/pérdidas con forma de cono. Algo similar ocurre cuando los precios de ejercicio de las opciones call y put difieren, en cuyo caso la gráfica de los beneficios/pérdidas adopta la forma de una cuna (strangle).

Lo mismo podemos decir de las estrategias butterfly y condor que dan lugar a una gráfica de los resultados posibles que nos recuerda la silueta de una mariposa o un cóndor, respectivamente.

\subsection{CARACTERÍSTICAS MORFOLÓGICAS DEL LÉXICO FINANCIERO}

Con respecto a las cuestiones de orden léxico y para dar cumplimiento al objetivo principal de este trabajo, enunciado en la Introducción, nos gustaría aportar las siguientes características de los términos financieros. Para ello, seguiremos el orden propuesto por Álvarez Calleja (2002). Esta clasificación constituirá el marco para la propuesta de traducción o complementación de algunos términos que no aparecen en los diccionarios especializados, con lo que cumpliremos los objetivos específicos marcados.

\subsection{TÉRMINOS PROCEDENTES DE OTRAS LENGUAS}

\begin{tabular}{|ll|}
\hline Término: & dinero legal. \\
Categoría gramatical: & adjetivo. \\
Marca de ponderación: & término normalizado. \\
Definición: & moneda emitida por un gobierno y que no tiene valor \\
& intrínseco. \\
Fuente de la definición: & Diccionario de la Real Academia Española (2001) [en \\
& línea]. Madrid: Espasa Calpe. http://www.rae.es/ \\
& {$[30 / 03 / 12]$} \\
\hline
\end{tabular}


(a) Términos procedentes del latín. Por ejemplo, término inglés: fiat money.

Otros ejemplos que se proponen:

- Price equilibrium $=$ precio de equilibrio.

- Denumerable $=$ numerable (Origen: $1560-70 ;<\mathrm{L}$ numeräbilis que se puede contar, equivale a numer(āre) numerar + -äbilis -able (véase www.dictionary.com/).

(b) Términos procedentes del griego. Por ejemplo, término inglés: stochastic.

\begin{tabular}{|ll|}
\hline Término: & estocástico. \\
Categoría gramatical: & adjetivo. \\
Marca de ponderación: & término normalizado. \\
Definición: & perteneciente o relativo al azar. \\
Fuente de la definición: & Diccionario de la Real Academia Española (2001) [en \\
& línea]. Madrid: Espasa Calpe. http://www.rae.es/ \\
& {$[30 / 03 / 12]$} \\
\hline
\end{tabular}

(c) Importante presencia de palabras de origen francés, como consecuencia lógica de los 400 años de dominio francés en Inglaterra. Por ejemplo, término inglés: arbitrage.

Término:

Categoría gramatical:

Marca de ponderación:

Definición:

Fuente de la definición: arbitraje.

sustantivo masculino.

término normalizado.

operación de cambio de valores mercantiles, en la que se busca la ganancia aprovechando la diferencia de precios entre unas plazas y otras.

Diccionario de la Real Academia Española (2001) [en línea]. Madrid: Espasa Calpe. http://www.rae.es/ [30/03/12]

\begin{tabular}{|ll|}
\hline Término: & numerario. \\
Categoría gramatical: & sustantivo masculino. \\
Marca de ponderación: & término normalizado. \\
Definición: & moneda acuñada, o dinero efectivo. \\
Fuente de la definición: & Diccionario de la Real Academia Española (2001) [en \\
& línea]. Madrid: Espasa Calpe. http://www.rae.es/ \\
& {$[30 / 03 / 12]$} \\
\hline
\end{tabular}


Lo mismo ocurre con el término inglés: arbitrageur, arbitrajista, aunque también existe el término shunter, de origen anglosajón, que significa corredor de bolsa que opera en dos mercados diferentes con un título en cotización en ambos.

\subsection{PALABRAS COMPUESTAS Y AFIJACIÓN: PREFIJOS Y SUFIJOS.}

Se trata de palabras formadas por dos o más morfemas léxicos (lexemas) que corresponden a una sola unidad. Por ejemplo, término inglés: lognormal.

Término:

Categoría gramatical: Marca de ponderación:

Definición:

Fuente de la definición: Diccionario de Ciencias de la Tierra. Madrid: Editorial Complutense, S.A., 2004.

Beigbeder Atienza, F. (1997). Diccionario Politécnico de las Lenguas Española e Inglesa. Madrid: Ediciones Díaz de Santos.

logarítmico normal, log-normal, geométrica.

adjetivo (va acompañando siempre a "distribución”).

término documentado en diccionarios.

una distribución en la que los logaritmos de los valores tienen una distribución gausiana (normal). Este tipo de distribución es común en el contexto de la geología; p. ej., los tamaños de partículas de sedimento.

En cuanto al uso de los prefijos, un ejemplo puede verse en el término inglés multiperiod.

\section{Término: multiperíodo.}

Categoría gramatical: adjetivo.

Marca de ponderación: término documentado en textos.

Contexto:

... a una inmunización multiperíodo, en la que el directivo ajusta el vencimiento de cada elemento a la corriente de responsabilidades...

Fuente del contexto: García Villalón, Julio y Martínez Barbeito, Josefina (2003).

Diccionario Técnico Inglés-Español Económico-Financiero-Actuarial. A Coruña: Netbiblo, S.L.

Por último, el uso de los sufijos se detecta en términos tales como riskless. 


\begin{tabular}{|ll|}
\hline Término: & sin riesgo, con certidumbre. \\
Categoría gramatical: & adjetivo. \\
Marca de ponderación: & término documentado en diccionarios. \\
Contexto: & riskeless or free asset: un activo cuyo rendimiento futuro es \\
& conocido hoy con certidumbre; riskless rate: el tanto ganado \\
& en una inversión sin riesgo; riskless rate of return: el tanto \\
& ganado en un activo sin riesgo. \\
& García Villalón, Julio y Martínez Barbeito, Josefina (2003). \\
& Diccionario Técnico Inglés-Español Económico-Financiero-Actuarial. \\
& A Coruña: Netbiblo, S.L. \\
\hline
\end{tabular}

Otros ejemplos:

- Payoff = beneficio.

\subsection{COLOCACIONES LÉXICAS.}

Se trata de unidades léxicas disjuntas que tienen un significado textual global y juntas expresan también una misma función. Las principales estructuras de los sustantivos compuestos pueden ser:

(a) Sustantivo + sustantivo. Por ejemplo, término inglés: state-price.

\begin{tabular}{|ll|}
\hline Término: & factor de descuento. \\
Categoría gramatical: & masculino. \\
Marca de ponderación: & término documentado en textos. \\
Contexto: & The existence of pricing rules, discount factors or state \\
& prices is crucial in the literature on capital markets. \\
Fuente del contexto: & Balbás, Alejandro y Jiménez-Guerra, Pedro (2009). \\
& Martingales and arbitrage: a new look. Rev. R. Acad. Cien. \\
& Serie A. Mat. Vol. 103 (2): 265-275. \\
\hline
\end{tabular}

Otros ejemplos que se proponen:

- Consumption process $=$ proceso consumo.

- Martingale measure = medida martingala.

- State-price deflator = function estocástica de descuento.

- Expiration time $=$ vencimiento.

- Propagation of information $=$ propagación de información (familia de subconjuntos $\mathfrak{I}_{n}$ ). 
- Information structure $=$ estructura de información (familia de subconjuntos $\mathfrak{I}_{n}$ ).

- State of the world = estado de la naturaleza.

- Security price = precio del activo (financiero).

- $\quad$ tock price $=$ precio de la acción.

(b) Adjetivo + sustantivo. Por ejemplo, término inglés: adapted process.

Término:

Categoría gramatical:

Marca de ponderación:

Definición:

Fuente de la definición: proceso adaptado.

masculino.

término documentado en textos.

Se dice que un proceso $X_{n}$ es adaptado a una filtración $\mathfrak{I}_{n}$ si la variable $X_{n}$ es $\mathfrak{J}_{n}$-medible, para cada $n \geq 1$.

Rincón, Luis: Introducción a los procesos estocásticos.

Departamento de Matemáticas. México DF: UNAM.

http://www.matematicas.unam.mx/lars [30/03/12].

Otros ejemplos que se proponen:

- Conditional expectation = esperanza condicionada.

- Marketed strategy = estrategia de mercado.

(c) Adjetivo + adjetivo. Por ejemplo, término inglés: short-lived.

\begin{tabular}{|ll|}
\hline Término: & a corto plazo. \\
Categoría gramatical: & unidad fraseológica. \\
Marca de ponderación: & término documentado en diccionarios. \\
Definición: & efímero, fugaz, pasajero. \\
Fuente de la definición: & Diccionario Oxford (1994). New York: Oxford University \\
& Press. \\
& WordReference.com English-Spanish Dictionary [en línea]. \\
& http://www.wordreference.com [30/03/12]. \\
\hline
\end{tabular}

\subsection{NEÓNIMOS}

Siguiendo a Rey (1988), para determinar si una unidad léxica es un neologismo, se tendrán en cuenta los siguientes parámetros:

- Diacronía: ha aparecido en un período reciente.

- Lexicografía: no aparece en los diccionarios.

- Inestabilidad sistemática: presenta inestabilidad formal o semántica.

- Psicología: los hablantes la perciben como una unidad nueva. 
Los neónimos tienen las siguientes características generales (Cabré, 1993):

- Surgen por necesidades denominativas y son muy estables en el tiempo. Por ejemplo, call option.

- Son reacios a la aparición de sinónimos, ya que estos pueden alterar su significado. Por ejemplo, contingent claim.

- Muchos son formas sintagmáticas. Por ejemplo, "mercados OTC (over the counter)".

- Habitualmente responden a una forma de composición culta. Por ejemplo, fiat money.

- Tienen una clara vocación internacional. Por ejemplo, martingale.

Este fenómeno ha dado lugar a la aparición de nuevos neologismos que se estudian de acuerdo con el siguiente esquema (Cabré, 1993):

- Si el literal del término refleja las características del mismo.

- Necesidad de abreviatura de los términos complejos.

- Adaptación fónica y gráfica a la lengua de destino.

- Posibilidad de producir derivados.

- Evitación de términos que desvirtúen el significado del término al que se pretende sustituir.

- Arraigo de su uso ya que, en caso afirmativo, se desaconseja el cambio de término.

- Etcétera.

A pesar de la inclusión de este epígrafe en el presente trabajo, hemos de decir que la presencia de neónimos en este artículo es puramente testimonial ya que el desarrollo de los mismos se señala como una posible línea futura de investigación.

\subsection{CARACTERÍSTICAS SINTÁCTICAS DEL LÉXICO FINANCIERO}

Con respecto a las cuestiones de orden sintáctico, nos gustaría aportar las siguientes características de los términos financieros:

(a) Uso del verbo may con significado de tiempo futuro En el siguiente texto, se puede observar el uso de este recurso ampliamente utilizado en los textos financieros:

Although a portfolio manager may have performed better than a benchmark, this does not necessarily mean that the portfolio manager satisfied the client's investment objective" (Fabozzi y Focardi, 2004: 9). Propuesta de traducción: “Aunque el 
gestor de una cartera hubiera obtenido mayor rendimiento que una cartera de referencia, esto no significa que necesariamente el gestor de la cartera haya satisfecho el objetivo de inversión del cliente.

(b) Uso frecuente de la forma no personal -ing. La forma -ing se puede traducir por:

- Gerundio: "Assuming that the risk of the portfolio was similar to that of the benchmark, it would appear that the manager outperformed the benchmark" (Fabozzi y Focardi, 2004: 9). Propuesta de traducción: "Suponiendo que el riesgo de la cartera fuera similar al de la cartera de referencia, figuraría que el gestor ha batido a la cartera de referencia".

- Infinitivo: "This step involves measuring the performance of the portfolio and then evaluating that performance relative to some benchmark" (Fabozzi y Focardi, 2004: 9). Propuesta de traducción: "Este paso conlleva medir la performance de la cartera y, a continuación, valorar la performance de alguna cartera de referencia".

- Sustantivo: "Selecting assets that intentionally cause the portfolio's risk profile to differ from that of the benchmark index is the way a manager actively manages a portfolio" (Fabozzi y Focardi, 2004: 9). Propuesta de traducción: "La selección de activos que deliberadamente hacen que el perfil de riesgo de la cartera se diferencie del de la cartera de referencia es la forma de que un agente gestione activamente una cartera". Otros ejemplos que se proponen:

- Arbitrage pricing $=$ precio del arbitraje.

- Stopping time $=$ momento de parada.

○ Trading strategy $=$ estrategia comercial.

(c) Frecuencia de la voz pasiva, que es necesario traducir por el se impersonal:

There are bond issues that have a provision granting the bond issuer an option to retire all or part of the issue prior to the stated maturity date. This feature is referred to as a call feature and a bond with this feature is said to be a callable bond". (Fabozzi y Focardi, 2004: 55). Propuesta de traducción: "Hay emisiones de bonos que tienen una provisión que concede al emisor de los bonos la opción de retirar todo o parte de la emisión antes de la 
fecha de vencimiento indicada. Este característica se conoce como una call feature y un bono con esta característica, se dice que es un callable bond.

(d) Uso restringido del determinante inglés. El artículo definido se usa cuando suponemos que el lector conoce el tema que se expone.

The additional margin deposited is called variation margin, and it is an amount necessary to bring the equity in the account back to its initial margin level". (Fabozzi y Focardi, 2004: 58). Propuesta de traducción: "El margen adicional depositado se llama margen de variación, y es el montante necesario para dejar la cuenta de capital en su nivel de margen inicial.

(e) El pronombre.

(e 1 Uso del demostrativo en algunos casos.

More recently, the diffusion of electronic transactions has made available a huge amount of empirical data. The availability of this data created the hope that economics could be given a more solid scientific grounding" (Fabozzi y Focardi, 2004: 76). Propuesta de traducción: "Más recientemente, la difusión de transacciones electrónicas ha hecho que una enorme cantidad de datos empíricos estén disponibles. La disponibilidad de estos datos creó la esperanza de que se le pudiera dar a la Economía una base científica más sólida.

( $\left.e_{2}\right)$ El demostrativo inglés puede ser traducido por un artículo u otro pronombre.

"This cost of borrowing can be incorporated into the model by reducing the yield on the asset" (Fabozzi y Focardi, 2004: 63). Propuesta de traducción: "Existe un coste inherente al préstamo que puede incorporarse al modelo reduciendo el rendimiento del activo".

\subsection{CARACTERÍSTICAS SEMÁNTICAS DEL LÉXICO FINANCIERO}

(a) La sinonimia. En una definición general, la sinonimia es aquella relación semántica entre significantes diferentes con significados semejantes, o 
ENTRECULTURAS Número 6. ISSN: 1989-5097. Fecha de publicación: 29-01-2014

aquellos elementos que tienen una comunidad de significado (sinonimia parcial). Por ejemplo, los términos ingleses normalize y standardize.

\begin{tabular}{|ll|}
\hline Término: & normalizar. \\
Categoría gramatical: & verbo. \\
Marca de ponderación: & término normalizado. \\
Definición: & tipificar (ajustar a un tipo o norma). \\
Fuente de la definición: & Diccionario de la Real Academia Española (2001) [en \\
& línea]. Madrid: Espasa Calpe. http://www.rae.es/ \\
& {$[30 / 03 / 12]$} \\
\hline
\end{tabular}

\begin{tabular}{|ll|}
\hline Término: & estandarizar. \\
Categoría gramatical: & verbo. \\
Marca de ponderación: & término normalizado. \\
Definición: & tipificar (ajustar a un tipo o norma). \\
Fuente de la definición: & Diccionario de la Real Academia Española (2001) [en \\
& línea]. Madrid: Espasa Calpe. http://www.rae.es/ \\
& {$[30 / 03 / 12]$} \\
\hline
\end{tabular}

(b) La polisemia. La polisemia es aquella relación semántica que se produce cuando un mismo significante puede tener diferentes significados.

(b1) La polisemia por deslizamiento de significado. Se trata de palabras que se utilizan en contextos diferentes, hasta que se va diferenciando su sentido y se llega a una especialización que lo hace totalmente diferente. Por ejemplo, el término inglés: liabilities.

\begin{tabular}{|ll|}
\hline Término: & responsabilidades. \\
Categoría gramatical: & femenino. \\
Marca de ponderación: & término normalizado. \\
Contexto: & La sociedad de responsabilidad limitada es la formada \\
& por reducido número de socios con derechos en \\
& proporción a las aportaciones de capital y en que solo se \\
& responde de las deudas por la cuantía del capital social. \\
& Diccionario de la Real Academia Española (2001) [en \\
Fuente del contexto: & línea]. Madrid: Espasa Calpe. http://www.rae.es/ \\
& {$[30 / 03 / 12]$} \\
Término: & pasivo. \\
Categoría gramatical: & masculino. \\
Marca de ponderación: & término normalizado. \\
\hline
\end{tabular}


ENTRECULTURAS Número 6. ISSN: 1989-5097. Fecha de publicación: 29-01-2014

\begin{tabular}{|ll|}
\hline Definición: & valor monetario total de las deudas y compromisos que \\
& gravan a una empresa, institución o individuo, y que se \\
& reflejan en su contabilidad. \\
Fuente de la definición: & Diccionario de la Real Academia Española (2001) [en \\
& línea]. Madrid: Espasa Calpe. http://www.rae.es/ \\
& {$[30 / 03 / 12]$}
\end{tabular}

(b2) La polisemia por lenguaje figurado. Se trata de palabras que ya no se asocian con el sentido original. Por ejemplo, el término inglés: replicate.

\begin{tabular}{|ll|}
\hline Término: & replicar. \\
Categoría gramatical: & verbo. \\
Marca de ponderación: & término documentado en textos. \\
Contexto: & si un fondo de inversión es el vehículo idóneo para un \\
& inversor que empieza, un fondo indexado es la alternativa \\
& más sencilla. Su objetivo es replicar la evolución de un \\
& índice determinado, lo que facilita la decisión del partícipe. \\
Fuente del contexto: & www.elpais.com/ [30/03/12] \\
\hline
\end{tabular}

Otros ejemplos que se proponen:

- Filtration $=$ filtración $\left(\right.$ familia de subconjuntos $\left.\mathfrak{I}_{n}\right)$.

- Exercise an option = ejercer una opción.

- Partition = partición.

- Refine $=$ refinar.

- Rank = rango (de una matriz).

- $\operatorname{Span}=$ espacio generado por ...

(b3) La polisemia por influencia extranjera. Se trata de palabras que ya no se asocian, por el motivo mencionado, con el sentido original. Por ejemplo, el término inglés: build.

\begin{tabular}{|ll|}
\hline Término: & construir. \\
Categoría gramatical: & verbo. \\
Marca de ponderación: & término documentado en textos. \\
Contexto: & Gestión pasiva es la desarrollada por un gestor que se \\
& limite a construir una cartera de activos que replique la \\
& composición del índice tomado como referencia. Una \\
& cartera de bolsa cuya composición sea idéntica, por \\
& ejemplo, a la del índice Ibex-35, tendrá sistemáticamente \\
& un rendimiento paralelo al de ese indicador. \\
\hline
\end{tabular}


ENTRECULTURAS Número 6. ISSN: 1989-5097. Fecha de publicación: 29-01-2014

\begin{tabular}{|lllll|}
\hline Fuente del contexto: & $\begin{array}{l}\text { Diccionario } \\
\text { http://www.finanzas.com }[30 / 03 / 12] .\end{array}$ & Finanzas.com & línea] \\
\hline
\end{tabular}

(c) La homonimia. La homonimia es la convergencia de significados diferentes en un mismo significante, o los mismos significantes con significados diferentes. De las tres vertientes de la homonimia léxica, la homografía (que es la que más nos interesa) se da con menor frecuencia que las otras dos (homonimia pura y homofonía). Véase De La Cruz Cabanillas (1998). Por tanto, podemos decir que, en el inglés de los términos financieros, apenas existe la homografía, por lo que no aportaremos ningún ejemplo.

(d) La hiponimia. La hiponimia es la pertenencia del significado de una palabra al significado más amplio de otra, lo que implica que puede establecerse jerarquías con las palabras. La hiponimia (relación de significados) se usa para delimitar un campo léxico. Así, dos términos hipónomos son, entre ellos, cohipónimos: tienen algo en común, son algo sinónimos. Por ejemplo, los términos ingleses: contingent claim, derivative instrument y option.

\begin{tabular}{|ll|}
\hline Término: & derecho contingente. \\
Categoría gramatical: & masculino. \\
Marca de ponderación: & término documentado en diccionarios. \\
Definición: & crédito eventual; título aleatorio, derecho (reclamación) que \\
& se puede realizar solamente si acaece uno o más resultados \\
& especificados. \\
Fuente de la definición: & García Villalón, Julio y Martínez Barbeito, Josefina (2003). \\
& Diccionario Técnico Inglés-Español Económico-Financiero-Actuarial. \\
& A Coruña: Netbiblo, S.L. \\
\hline
\end{tabular}

Término:

Categoría gramatical:

Marca de ponderación:

Contexto:

Fuente del contexto: instrumento derivado.

masculino.

término documentado en diccionarios.

derivado, activo derivado, producto derivado.

Castelo Montero, Miguel (2003). Diccionario Comentado de Términos Financieros Ingleses de Uso Frecuente en Español. A Coruña: Netbiblo, S.L.

\section{Término:}

Categoría gramatical:

Marca de ponderación:

Definición: opción.

masculino.

término normalizado.

derecho a comprar o vender algo en plazo y precio previamente acordados. 
ENTRECULTURAS Número 6. ISSN: 1989-5097. Fecha de publicación: 29-01-2014

Fuente de la definición: Diccionario de la Real Academia Española (2001) [en línea]. Madrid: Espasa Calpe. http://www.rae.es/ $[30 / 03 / 12]$.

La jerarquía que se establece entre estos tres términos es la siguiente (el símbolo $\subset$ significa “incluido en"):

\section{Option $\subset$ Derivative instrument $\subset$ Contingent claim.}

En efecto, el concepto de derivative instrument incluye las opciones (options) y los futuros, entre otros, mientras que el término contingent claim incluye, aparte de los instrumentos derivados, otros muchos productos pertenecientes al Sector de los Seguros (seguros de vida, seguros de ahorro, seguros mixtos, unit linked, etc.).

(e) La antonimia. La antonimia es la oposición semántica de dos términos. Esta oposición en el significado puede darse, además, en la forma léxica.

\begin{tabular}{|l|l|}
\hline \multicolumn{1}{|c|}{ Término } & \multicolumn{1}{c|}{ Antónimo } \\
\hline \multirow{2}{*}{ Arbitrage oportunity } & Absence of arbitrage \\
\cline { 2 - 2 } & Arbitrage free \\
\hline Continuous-time setting & Discrete-time setting \\
\hline Continuous-state setting & Discrete-state setting \\
\hline One-period setting & Multiperiod setting \\
\hline \multirow{3}{*}{ Risky } & Risk-neutral \\
\cline { 2 - 2 } & Riskless \\
\cline { 2 - 2 } & Risk free \\
\hline European option & American option \\
\hline Path dependence & Path independence \\
\hline Complete market & Incomplete market \\
\hline
\end{tabular}

(f) Los falsos amigos. Son aquellos términos de la lengua fuente y de la lengua término que tienen significantes similares pero que tienen significados distintos.

\begin{tabular}{|l|l|l|}
\hline \multicolumn{1}{|c|}{ Término } & \multicolumn{1}{c|}{ Falso amigo } & \multicolumn{1}{c|}{ Equivalente correcto } \\
\hline Commodity & Comodidad & Mercadería, materia prima \\
\hline Expectation & Expectación & Esperanza \\
\hline Gross return & Retorno grueso & Beneficio bruto \\
\hline
\end{tabular}


(g) La prosodia o asociación semántica. La prosodia semántica describe la forma en que algunas palabras, aparentemente neutras, pueden ser consideradas positivas o negativas por medio de asociaciones que ocurren frecuentemente con colocaciones particulares.

Por ejemplo, la palabra red posee una prosodia semántica negativa ya que sus colocaciones más típicas en Finanzas son red interest (números rojos en una cuenta corriente) o in the red (color de la cotización a la baja de un título cuando predominan las órdenes de venta).

La prosodia semántica en el contexto del Inglés Empresarial nos permite describir con mayor claridad las relaciones entre las palabras utilizadas en el contexto empresarial. En Nelson (2006: 217-234) se ha demostrado que el léxico empresarial muestra una tendencia para colocarse con conjuntos prosódico-semánticos recurrentes pero también conserva el potencial de colocación con palabras individuales. El análisis demostró que el mundo empresarial gira alrededor de conjuntos semánticos recurrentes: personas, instituciones, lugares y dinero, por ejemplo.

Por último, existe mucha evidencia que demuestra que las palabras que se encuentran en el contexto del Inglés Empresarial pueden asociarse con prosodias semánticas específicas de la empresa, pero también esas palabras pueden asociarse con grupos prosódico-semánticos del inglés general.

\subsection{LA METÁFORA EN LOS TEXTOS FINANCIEROS}

A pesar de que la metáfora constituye también un proceso semántico en la formación de términos financieros, las razones por las que le hemos dedicado un epígrafe aparte en la traducción de los textos financieros son dos:

1. El estudio de la metáfora en los textos económicos y financieros constituye per se un tema propio de investigación como puede observarse en la literatura especializada. Para un estudio exhaustivo del uso de la metáfora en el inglés empresarial, puede consultarse a Cortés de los Ríos (2000) o a Cruz Martínez (2002: 179-200).

2. La enorme importancia de esta figura retórica en los textos sobre informes financieros y su rápida incorporación en el estilo de los artículos académicos.

En Charteris-Black y Ennis (2001: 249-266) se define una metáfora como el fenómeno lingüístico por el cual una palabra o expresión se utiliza con un sentido distinto al que tiene en otros contextos. Un buen ejemplo de metáforas lo constituye el caso de palabras que normalmente se asocian a 
fenómenos físicos pero que se aplican también a fenómenos mentales abstractos. Por ejemplo, las palabras volatilidad (volatility) y burbuja (bubble) se utilizan para describir el comportamiento del mercado financiero y también para describir el comportamiento de un gas. Por otra parte, Lakoff y Johnson (1980), citados por Charteris-Black y Ennis (2001, op. cit.), utilizan el término "metáfora conceptual" para referirse a la representación que describe cómo dos palabras o expresiones procedentes de dominios aparentemente diferentes, pueden asociarse a un nivel cognitivo subyacente. Por ejemplo, las expresiones putting down roots y going to seed (echar raíces), procedentes del mundo de las plantas y de las personas se aplican indistintamente para expresar la idea.

Por lo que respecta a los textos económicos, en general, varios autores han señalado que son "muy metafóricos". Esta afirmación se justifica por la frecuencia con que emplean algunos términos tales como buman capital (capital humano), falling unemployment (caída del desempleo), demand expansion and contraction (expansión y contracción de la demanda), credit flows (flujos de crédito) o liquidity squeeze (recorte de liquidez). Por tanto y de acuerdo con lo anterior, se hace necesario un análisis de las formas superficiales y las metáforas de base conceptual pertenecientes a la Economía y a los procesos económicos. En este sentido, podemos encontrar un cierto número de casos en los que la similitud de significado se debe a un origen común del latín.

A pesar de la innegable existencia de metáforas en los textos contenidos en informes financieros, en el corpus utilizado en este trabajo, hemos podido identificar sólo los siguientes: confidence, jump, bubble, volatile, behaviour, rise, oscillate, fluctuation y volatility, lo que representa un porcentaje muy pequeño con respecto a los que pueden aparecer en los informes financieros.

\section{CONCLUSIONES}

La traducción de términos financieros presenta una serie de características propias que la diferencian de la traducción de otras ramas del saber. Estas características han sido descritas a lo largo de este artículo e ilustradas con múltiples ejemplos. No obstante, como apuntábamos al principio, hemos detectado que existe una laguna en la traducción de algunos términos financieros, concretamente los que se sitúan en la intersección de la Economía, las Matemáticas y las Finanzas. En efecto, a partir de la consulta de diccionarios especializados, hemos podido constatar que este déficit se manifiesta en un doble sentido. En primer lugar, existen muchos términos financieros que no están referenciados en los diccionarios especializados, y, en segundo lugar, existen términos que, aún estando referenciados, presentan una información insuficiente para las necesidades de un traductor. Por ejemplo, 
términos tales como riskless, martingale o short-lived son relativamente frecuentes en ciertos textos financieros, pero no se encuentran en los diccionarios especializados. Por otra parte, el traductor debe conocer que otros términos como straddle (cono), strange (cuna), butterfly (mariposa) y condor (cóndor) tienen su origen en la similitud que, con un poco de imaginación, puede establecerse entre las gráficas de los posibles beneficios de estrategias combinadas con opciones call y put, y la forma (silueta) de estos animales u objetos.

Después de llevar a cabo un muestreo para seleccionar los términos financieros más adecuados para este trabajo, hemos aplicado el modelo propuesto por Cabré y Tebé (2005: 19-41) para tratar de elaborar un trabajo terminológico de "enriquecimiento lingüístico" que pueda ser aplicado por los traductores en la confluencia de las áreas citadas anteriormente. Este enriquecimiento no sólo debe tener consecuencias metodológicas sino que debe servir de ayuda para que el traductor pueda llevar a cabo un proceso de inmersión en el texto financiero. En otras palabras, el traductor no sólo ha de ser capaz de llevar a cabo una traducción fiel sino que, además, debe estar capacitado para adentrarse en el contenido del texto y llevar a cabo así propuestas de mejora y adecuación de la propia redacción original.

Finalmente, quedaría por establecer una relación entre los dos objetivos básicos de este trabajo, a saber, la traducción de determinados términos financieros y la necesidad de información complementaria acerca ellos. Si tenemos en cuenta la correspondencia biunívoca entre término y concepto, en muchas ocasiones, es muy difícil encontrar una traducción adecuada para un término de las Finanzas. Éste sería el caso del término state-price que aquí se ha traducido como "factor de descuento". Esta dificultad podría determinar una mayor o menor predisposición de los usuarios de estos términos a sustituir o no la voz anglosajona por su(s) correspondiente(s) equivalente(s) en español. Así, el término swap podría ser un buen ejemplo de término con cierta resistencia a sustituirse por su traducción. Dicho grado de predisposición nos anima a emprender un futuro trabajo para el que, entre otras herramientas, elaboraríamos una encuesta dirigida a los profesores universitarios e investigadores en Finanzas de España e Hispanoamérica.

\section{BIBLIOGRAFÍA}

BIBLIOGRAFÍA SOBRE TERMINOLOGÍA Y TRADUCCIÓN

ÁLVAREZ CALLEJA, M. A. (2002). Traducción Jurídica Inglés-Español. Madrid: Universidad Nacional de Educación a Distancia. 
CABRÉ CASTELLVÍ, M. T. (1993). La Terminología. Teoría, metodología, aplicaciones. Barcelona: Editorial Antártida / Empúries.

CABRÉ CASTELLVÍ, M. T. y C. Tebé (2005). "El trabajo terminológico multilingüe de enriquecimiento lingüístico: una propuesta metodológica”. Revista Española de Lingüistica Aplicada, Vol. Extra 1 (Ejemplar dedicado a: Lingüística): 19-41.

CABRÉ CASTELLVÍ, M. T. (2008a). "El principio de poliedricidad: la articulación de lo discursivo, lo cognitivo y lo lingüístico en Terminología (I)". Ibérica 1(16): 9-36.

CABRÉ CASTELLVÍ, M. T. (2008b). "La terminologia: desenvolupament i utilitat en la formació de traductors" [en línea]. Tradumàtica, 6: http://www.fti.uab.es/tradumatica/revista/num6/articles/01/art.htm

CHARTERIS-BLACK, J. \& T. Ennis (2001). "A comparative study of metaphor in Spanish and English financial reporting”. English for Specific Purposes 20: 249-266.

CORTÉS DE LOS RÍOS, M. E. (2000). Análisis cognitivo-axiológico del discurso publicitario en la prensa económico-empresarial en lengua inglesa. Almería: Servicio de Publicaciones de la Universidad de Almería.

CRUZ MARTÍNEZ, M. S. (2002). "La semántica en el inglés profesional y académico". RESLA 15: 179-200.

DE LA CRUZ CABANILLAS, I. (1998). "Aproximación metodológica a la homonimia léxica en inglés". Revista de investigación e innovación en la clase de idiomas 10: 43-52.

DÍAZ HORMiGO, M. T. (2007). "Aproximación lingüística a la neología léxica”. En Martín Camacho, José Carlos y Rodríguez Ponce, María Isabel (eds.). Morfología: investigación, docencia, aplicaciones. Actas del II Encuentro de Morfología: investigación y docencia. Servicio de Publicaciones de la Universidad de Extremadura: 33-54.

FIJO LEÓN, M. I. (2007). "La enseñanza de la terminología a los alumnos de Traducción e Interpretación: el caso de la Universidad Pablo de Olavide”. Panace@8 (26):193-198.

FUERTES-OLIVERA, P. A. y S. Gómez-Martínez (2004). "Empirical assessment of some learning factors affecting Spanish students of Business English". English for Specific Purposes 23: 163-180.

HERNÁNDEZ GUERRA, C. y J. M. Hernández Guerra (2008). “Discoursive analysis and pragmatic metadiscourse in four sub-areas of Economics research articles". Ibérica 16: 81-108.

LAKOFF G. Y M. JONSON (1980). Metáfora de la vida cotidiana. Madrid: Cátedra. 
NEFF VAN AERTSELAER, J.A. (2006). "A rhetorical analysis approach to English for Academic Purposes". Revista de Lingüistica y Lenguas Aplicadas 1: 63-72.

NELSON, M. (2006). "Semantic associations in Business English: A corpusbased analysis". English for Specific Purposes 25: 217-234.

REY, A. (1988). "Dictionnaire et néologie". En: Actes du colloque "Terminologie et technologies nowvelles".

SUAU JIMÉNEZ, F. (1999). "El género y el registro aplicados a la traducción del discurso profesional en lengua inglesa: un enfoque funcional". En Aleza, M.; Fuster, M. y Lépinette, B. (eds.). El Contacto Lingüístico en el Desarrollo de las Lenguas Occidentales. Quaderns de Filologia, Vol. IV. Universitat de València.

\section{BIBLIOGRAFÍA DE FINANZAS}

BALBÁS, A. Y JIMÉNEZ-GUERRA, P. (2009). "Martingales and arbitrage: a new look". Rev. R. Acad. Cien. Serie A. Mat. 103 (2): 265-275.

COSTA RAN, L. Y FONT VILALTA, M. (1992). Nuevos Instrumentos Financieros en la Estrategia Empresarial. Madrid: Editorial ESIC.

FABOZZI, F.J. AND FOCARDI, S.M. (2004). The Mathematics of Financial Modeling and Investment Management. New Jersey: John Wiley \& Sons.

HULL, J.C. (2002). Introducción a los Mercados de Futuros y Opciones. $4^{a}$ edición. Madrid: Ed. Prentice-Hall.

LAMOTHE, P. (1993). Opciones Financieras. Un enfoque Fundamental. Madrid: Editorial McGraw-Hill.

RINCÓN, L. "Introducción a los procesos estocásticos". Departamento de Matemáticas. México DF: UNAM. http://www.matematicas.unam.mx/lars [30/03/12].

\section{DICCIONARIOS}

ALCARAZ VARÓ, E. Y HUGHES, B. (2008). Diccionario de Términos Económicos, Financieros y Comerciales. Barcelona: Editorial Ariel.

BEIGBEDER ATIENZA, F. (1997). Diccionario Politécnico de las Lenguas Española e Inglesa. Madrid: Ediciones Díaz de Santos, S.A.

CASTELO MONTERO, M. (2003). Diccionario Comentado de Términos Financieros

Ingleses de Uso Frecuente en Español. A Coruña: Netbiblo, S.L.

Diccionario de Ciencias de la Tierra. Madrid: Editorial Complutense, S.A., 2004. 
Diccionario de la Real Academia Española 2001 [en línea]. Madrid: Espasa Calpe. http://www.rae.es/ [30/03/12].

Diccionario Finanzas.com [en línea] http://www.finanzas.com [30/03/12].

Diccionario Oxford 1994. New York: Oxford University Press.

Lo esencial. Finanzas 1994. Madrid.

Oxford Advanced Learner's Dictionary of Current English 1995. Oxford University Press.

WordReference.com English-Spanish Dictionary [en línea].

http://www.wordreference.com [30/03/12].

GARCÍA VILLALÓN, J. Y MARTÍNEZ BARBEITO, J. (2003). Diccionario

Técnico Inglés-Español Económico-Financiero-Actuarial. A Coruña: Netbiblo, S.L. www.elpais.com/ $[30 / 03 / 12]$. 